\title{
Josephson Network as a Model for Inhomogeneous Superconductor: a Microwave Power Absorption
}

\author{
A. Rycerz AND J. SpaleK \\ Instytut Fizyki im. Mariana Smoluchowskiego \\ Uniwersytet Jagielloński, Reymonta 4, 30-059 Kraków, Poland \\ We discuss the applied magnetic field dependence of the microwave \\ absorption by a three-dimensional array of up to $30 \times 30 \times 30$ Josephson \\ junctions with random parameters including the resistivity, capacity, and \\ inductance of each junction. The numerical simulation results for the net- \\ works show characteristic microwave absorption anomalies observed in the \\ ceramic samples of high temperature superconductor $\mathrm{YBa}_{2} \mathrm{Cu}_{3} \mathrm{O}_{7-x}$. We \\ also provide a discussion of the absorption in simple analytical terms of the \\ Josephson loop instabilities.
}

PACS numbers: 74.81.Fa, 74.25.Nf

\section{Introduction}

Soon after the discovery of high temperature superconductivity it has been established [1-3] that in ceramic samples of $\mathrm{YBa}_{2} \mathrm{Cu}_{3} \mathrm{O}_{7-x}$ there exists a large nonresonant microwave absorption in zero and low applied magnetic field that can be associated with the transition to the superconducting phase. An exemplary field dependence of this absorption is displayed in Fig. 1 [1]. A broad absorption structure is modulated by a noisy signal with a clear evidence of a quasiperiodic substructure. One can also observe nonlinear phenomena, e.g. a generation of odd harmonics in zero applied field and of even harmonics in nonzero field [4].

The experimental setup used to measure the spectrum depicted in Fig. 1 [1] is an EPR spectrometer, which provides the magnetic resonance response due to $\mathrm{Cu}^{2+}$ ions in the normal phase in the field range of $0.35 \div 0.4 \mathrm{~T}$. The lowerfield signal has a reversed phase with respect to the usual (Lorentzian) EPR line. Therefore, this low-field magnetically-modulated microwave absorption (MMMA) has been attributed to the power loss in current loops connected by the dissipative Josephson junctions, which in turn are created between the grains in a ceramic sample. Actually, such junctions appear also in the monocrystalline sample [5], 


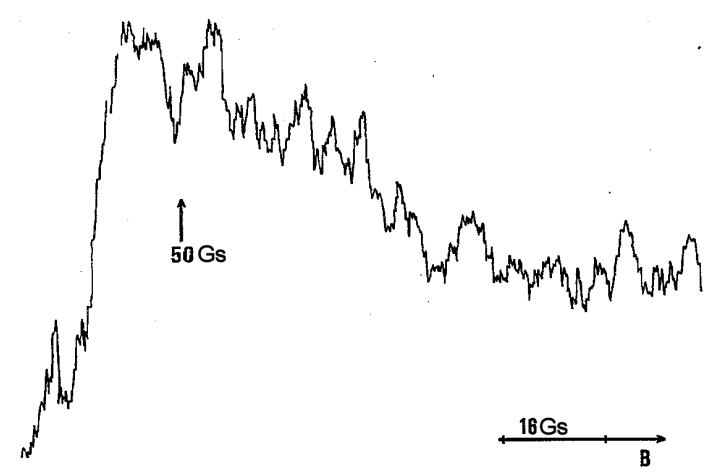

Fig. 1. First derivative of the power MMMA spectrum showing the 50 Gs peak for sample of $\mathrm{YBa}_{2} \mathrm{Cu}_{3} \mathrm{O}_{6+x}$ at $T=80 \mathrm{~K}$. Reprinted from Ref. [1].

although their presence in good quality untwinned monocrystalline samples should be associated with the Cooper-pair tunneling along the $c$-axis.

Because of the potential application of ceramic high temperature superconductors in various microwave applications, it is important to study the properties of a general three-dimensional array of the Josephson junctions shunted by resistance $(R)$, capacitance $(C)$, and with inductance $(L)$, together with inclusion of some random variations of the junction parameters. Such model can be called the disordered $R L C$-SJJ model [6]. For that purpose we consider a lattice depicted in Fig. 2, where $\theta_{i j k}^{\alpha}$ labels the phase difference in the direction $\alpha=x, y, z$, and $(i, j, k)$ locate the junction position in the three-dimensional array. This model was considered in various simplified situations by a number of authors [7]. Here we consider a 3-dimensional granular structure and disregard the circumstance that the grains themselves may have a coupled Josephson structure along the $c$-axis, perpendicular to the $\mathrm{CuO}_{2}$ planes [8]. In other words, the intergranular critical currents are assumed to be much smaller than their intragranular counterpart. This should allow us to address the question about the role of the Josephson network in causing the hysteresis while cycling slowly the applied field, as well as the question of the existence of a residual contribution in the zero field.

\section{The model}

\subsection{Dissipative lattice of the Josephson junctions}

We start from the gauge invariant phase difference $\theta$ between points 1 and 2 :

$$
\theta_{12} \equiv \int_{1}^{2} \mathrm{~d} \boldsymbol{l} \cdot\left(\nabla \phi-\frac{2 \pi}{\Phi_{0}} \boldsymbol{A}\right)
$$

where $\nabla \phi$ is the gradient of the phase of macroscopic wave function, $\boldsymbol{A}$ is the vector potential, and the integration takes place between the two sides of the junction with the sign convention in accordance with the coordinate directions shown in 


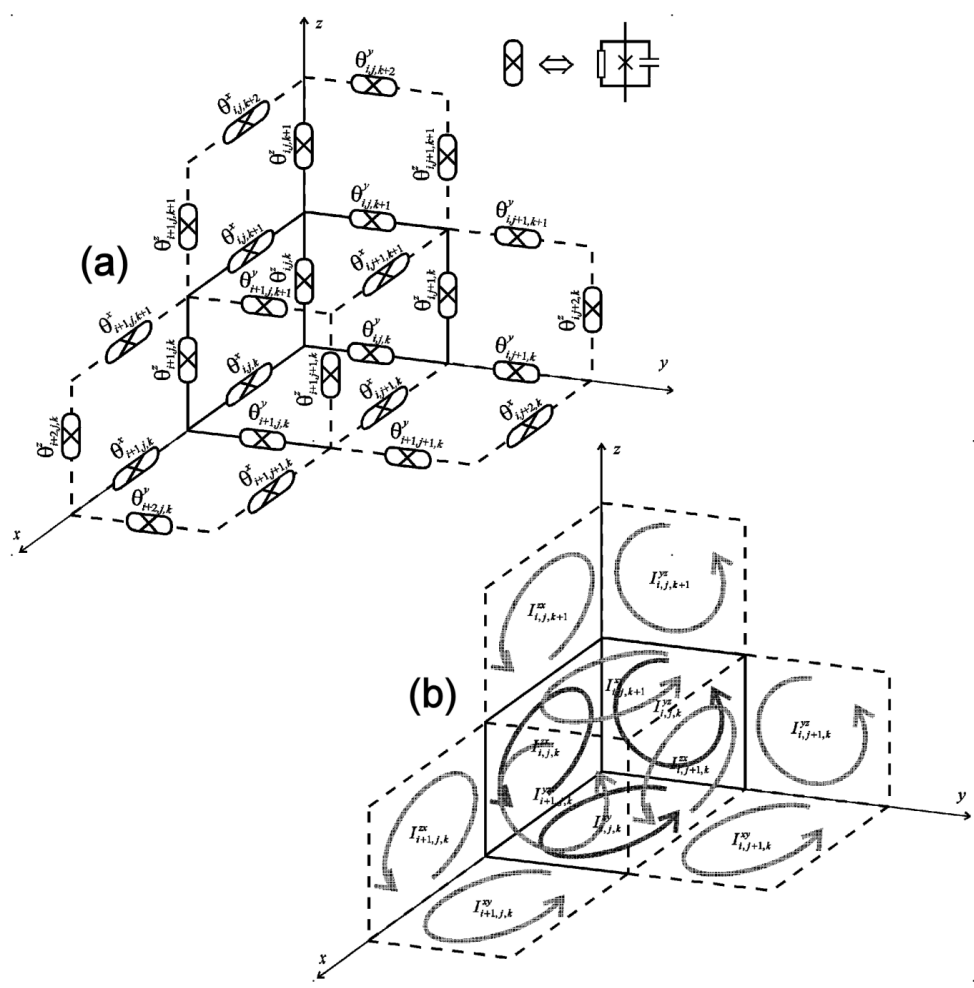

Fig. 2. Josephson network: (a) gauge invariant phases $\theta^{\alpha}$ on individual junctions, (b) the current distribution in loops composing the lattice.

Fig. 2a. Taking into account the single valuedness of the wave function, the Stokes theorem for the elementary loop, we obtain the relation between e.g. the magnetic flux piercing the loop in $x y$ plane and the phase differences on the Josephson junctions composing the loop in the form

$$
\frac{2 \pi}{\Phi_{0}} \Phi_{i, j, k}^{x y}=-\left(\theta_{i, j, k}^{x}-\theta_{i, j+1, k}^{x}+\theta_{i+1, j, k}^{y}-\theta_{i, j, k}^{y}\right) .
$$

For a convenience, we label the loops currents $I_{i j k}^{\alpha \tilde{L}}$ in the same manner as the fluxes $\Phi_{i j k}^{\alpha \tilde{L}}$, as shown explicitly in Fig. $2 \mathrm{~b}$.

Introducing reduced quantities $\tilde{\Phi}=\Phi / \Phi_{0}$, and $\tilde{\theta}=\theta / 2 \pi$ we can write down the relation (2) for each plane $\alpha \tilde{L}=x y, y z$, and $z x$ with the replacement $\Phi \rightarrow \tilde{\Phi}$ and $\theta \rightarrow \tilde{\theta}$, so that

$$
\begin{aligned}
& \tilde{\Phi}_{i, j, k}^{x y}=-\left(\tilde{\theta}_{i, j, k}^{x}+\tilde{\theta}_{i+1, j, k}^{y}-\tilde{\theta}_{i, j+1, k}^{x}-\tilde{\theta}_{i, j, k}^{y}\right), \\
& \tilde{\Phi}_{i, j, k}^{z x}=-\left(\tilde{\theta}_{i, j, k}^{z}+\tilde{\theta}_{i, j, k+1}^{x}-\tilde{\theta}_{i+1, j, k}^{z}-\tilde{\theta}_{i, j, k}^{x}\right), \\
& \tilde{\Phi}_{i, j, k}^{y z}=-\left(\tilde{\theta}_{i, j, k}^{y}+\tilde{\theta}_{i, j+1, k}^{z}-\tilde{\theta}_{i, j, k+1}^{y}-\tilde{\theta}_{i, j, k}^{z}\right) .
\end{aligned}
$$


The cyclic properties of these relations are easy to grasp. The $(i, j, k)$ take the following values for the cubic array containing $N \times N \times N$ junctions:

$$
\begin{gathered}
\tilde{\Phi}_{i, j, k}^{x y}: \quad i=0, \ldots, N-1 ; \quad j=0, \ldots, N-1 ; \quad k=0, \ldots, N \\
\tilde{\Phi}_{i, j, k}^{z x}: \quad i=0, \ldots, N-1 ; \quad j=0, \ldots, N ; \quad k=0, \ldots, N-1 ; \\
\tilde{\Phi}_{i, j, k}^{y z}: \quad i=0, \ldots, N ; \quad j=0, \ldots, N-1 ; \quad k=0, \ldots, N-1 .
\end{gathered}
$$

The corresponding relation for the junction phase differences are

$$
\begin{gathered}
\tilde{\theta}_{i, j, k}^{x}: \quad i=0, \ldots, N-1 ; \quad j=0, \ldots, N ; \quad k=0, \ldots, N ; \\
\tilde{\theta}_{i, j, k}^{y}: \quad i=0, \ldots, N ; \quad j=0, \ldots, N-1 ; \quad k=0, \ldots, N ; \\
\tilde{\theta}_{i, j, k}^{z}: \quad i=0, \ldots, N ; \quad j=0, \ldots, N ; \quad k=0, \ldots, N-1 .
\end{gathered}
$$

Let us write down the dynamic equation for the current in individual loop. For a single $R C$ shunted Josephson junction (cf. the inset in Fig. 2a) the total current $I$ through it is composed of 3 terms, namely

$$
I=C \frac{\Phi_{0}}{2 \pi} \ddot{\theta}+\frac{1}{R} \frac{\Phi_{0}}{2 \pi} \dot{\theta}+I_{\mathrm{c}} \sin \theta
$$

where $C$ and $R$ are the junction capacity and resistance, respectively and $I_{\mathrm{c}}$ is its critical current (the self-inductance $L$ is included through the relation to the flux, $\Phi=-L I$, see below). In the case of a junction placed on $z$-axis inside the network, the current through it is composed of four contributions from the loops attached to it, namely

$$
C \frac{\Phi_{0}}{2 \pi} \ddot{\theta}_{i, j, k}^{z}+\frac{1}{R} \frac{\Phi_{0}}{2 \pi} \dot{\theta}_{i, j, k}^{z}+I_{\mathrm{c}} \sin \theta_{i, j, k}^{z}=I_{i, j, k}^{z x}-I_{i-1, j, k}^{z x}-I_{i, j, k}^{y z}+I_{i, j-1, k}^{y z} .
$$

Defining additional dimensionless variables

$$
\begin{gathered}
\tilde{I} \equiv I / I_{\mathrm{c}}, \quad \tilde{L} \equiv L I_{\mathrm{c}} / \Phi_{0}, \quad \tilde{\Gamma} \equiv \sqrt{L C} / R C, \\
\tilde{t} \equiv t / \sqrt{L C}, \quad \dot{\tilde{\theta}} \equiv \mathrm{d} \tilde{\theta} / \mathrm{d} \tilde{t}, \quad \ddot{\tilde{\theta}} \equiv \mathrm{d}^{2} \tilde{\theta} / \mathrm{d} \tilde{t}^{2},
\end{gathered}
$$

we can write down a complete set of dynamic equations for $\tilde{\theta}^{x, y, z}$ in the form

$$
\begin{aligned}
& \ddot{\tilde{\theta}}_{i, j, k}^{x}+\tilde{\Gamma} \dot{\tilde{\theta}}_{i, j, k}^{x}+\tilde{L} \sin 2 \pi \tilde{\theta}_{i, j, k}^{x}=\tilde{L}\left(\tilde{I}_{i, j, k}^{x y}-\tilde{I}_{i, j-1, k}^{x y}-\tilde{I}_{i, j, k}^{z x}+\tilde{I}_{i, j, k-1}^{z x}\right), \\
& \ddot{\tilde{\theta}}_{i, j, k}^{y}+\tilde{\Gamma} \dot{\tilde{\theta}}_{i, j, k}^{y}+\tilde{L} \sin 2 \pi \tilde{\theta}_{i, j, k}^{y}=\tilde{L}\left(\tilde{I}_{i, j, k}^{y z}-\tilde{I}_{i, j, k-1}^{y z}-\tilde{I}_{i, j, k}^{x y}+\tilde{I}_{i-1, j, k}^{x y}\right), \\
& \ddot{\tilde{\theta}}_{i, j, k}^{z}+\tilde{\Gamma}_{i, j, k}^{z}+\tilde{L} \sin 2 \pi \tilde{\theta}_{i, j, k}^{z}=\tilde{L}\left(\tilde{I}_{i, j, k}^{z x}-\tilde{I}_{i-1, j, k}^{z x}-\tilde{I}_{i, j, k}^{y z}+\tilde{I}_{i, j-1, k}^{y z}\right) .
\end{aligned}
$$

The boundary conditions are set as

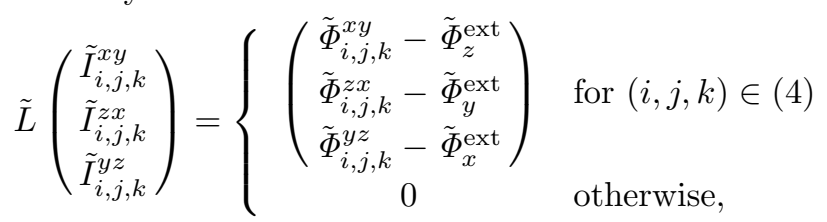


where $\tilde{\Phi}_{\alpha}^{\text {ext }}$ is the external flux (in units $\Phi_{0}$ ) enclosed by the plane perpendicular to the axis $\alpha=x, y, z$. Thus we see that the external flux is screened by the self-inductance and Josephson supercurrents at the surface; these currents ignite internal supercurrents distribution. Substituting the expression for the current (9) via fluxes (10), and subsequently, using the relation (3) between the fluxes and phases, we arrive at the closed system of $N_{J} \equiv 3 N(N+1)^{2}$ equations for $\tilde{\theta}_{i, j, k}^{\alpha}(t)$ of a homogeneous Josephson array, whose solution will be discussed first numerically, and then in physical terms. For that purpose we have to introduce first the random variations of the junction parameters to make the situation more realistic.

\subsection{Inclusion of the grain size distribution and the numerical procedure}

The system of nonlinear Eqs. (3), (9) and (10) is solved numerically and, subsequently, analyzed in qualitative terms. The physical situation is reflected by assuming that we have a microwave field of period $T$ and amplitude $A_{\mathrm{m}}$ applied along the $x$-axis and $y$-axis and a static magnetic field applied in the $z$-direction. In other words, the external magnetic flux piercing the network has the components

$$
\tilde{\Phi}_{x}^{\operatorname{ext}}(\tilde{t})=A_{\mathrm{m}} \sin \left(\frac{2 \pi \tilde{t}}{T}\right), \quad \tilde{\Phi}_{y}^{\operatorname{ext}}(\tilde{t})=0, \quad \tilde{\Phi}_{z}^{\mathrm{ext}}(\tilde{t})=\alpha \tilde{t},
$$

where $\alpha \ll 1$ describes the slow sweeping rate of the applied (quasistatic) field.

The description of real systems requires taking into account the random variation of the parameters of each loop in the array. For this purpose we introduce coefficients $a_{i, j, k}^{x y}, a_{i, j, k}^{y z}$, and $a_{i, j, k}^{z x}$, which characterize the size of elementary loop with coordinates $(i, j, k)$. These parameters scale the magnetic flux $\tilde{\Phi}_{x}^{\text {ext }}$ in the following way: $\tilde{\Phi}_{x}^{\text {ext }}(\tilde{t}) \rightarrow \tilde{\Phi}_{x}^{\text {ext }}(\tilde{t}) / a_{i, j, k}^{y z}$, and in a similar manner the other components. Analogously, the junction parameters $\tilde{L}$ and $\tilde{\Gamma}$ fluctuate and depend on the direction, e.g. $\tilde{\Gamma} \rightarrow \tilde{\Gamma}_{i, j, k}^{x}, \tilde{L} \rightarrow \tilde{L}_{i, j, k}^{y z}$, etc. All the parameters fluctuate according to the Gaussian distribution with $10 \%$ dispersion to match the experimentally estimated dispersion of crystallite size [1] around the values $a=a_{0} \approx 1$ (corresponding to the average grain size $d=0.74 \mu \mathrm{m}$ ), $\tilde{L}_{0}=1$, and $\tilde{\Gamma}_{0}=5$ (overdamped regime). The representative values of all the parameters in both physical and dimensionless units (used in the simulation) are provided in Ref. [9]. In particular, the microwave field period $\tilde{T}=50$ corresponds to the frequency much higher than that in the experiments [1-3] $(9.4 \mathrm{GHz})$. The higher frequency was taken to accelerate the computations and we do not expect any essential influence of this factor on the output character.

The system of $N_{J}$ differential equations, generated for $N=1 \div 30$ was solved numerically using the fourth-order Runge-Kutta method [10] taking randomly initial values of $\tilde{\theta}(0)$ and $\dot{\tilde{\theta}}(0)$, as well as setting the time step $\Delta \tilde{t}=0.1$. The sweeping rate of the quasistatic field was $\alpha=10^{-3}$ and in the range $[0,10]$. Typically, the solution simulation consisted of $10^{5}$ time steps. 


\section{Analysis of the results}

\subsection{Numerical solution}

The power absorbed (per one junction) can be written in dimensionless units as

$$
\tilde{P}=\frac{1}{N_{J}}\left\langle\sum_{i, j, k} \tilde{\Gamma}_{i, j, k}^{x}\left(\dot{\tilde{\theta}}_{i, j, k}^{x}\right)^{2}+\tilde{\Gamma}_{i, j, k}^{y}\left(\dot{\tilde{\theta}}_{i, j, k}^{y}\right)^{2}+\tilde{\Gamma}_{i, j, k}^{z}\left(\dot{\tilde{\theta}}_{i, j, k}^{z}\right)^{2}\right\rangle
$$

where averaging takes place over the time interval equal to the microwave field period $\tilde{T}$. This expression corresponds to the expression $P=U^{2} / R$ in dimen-

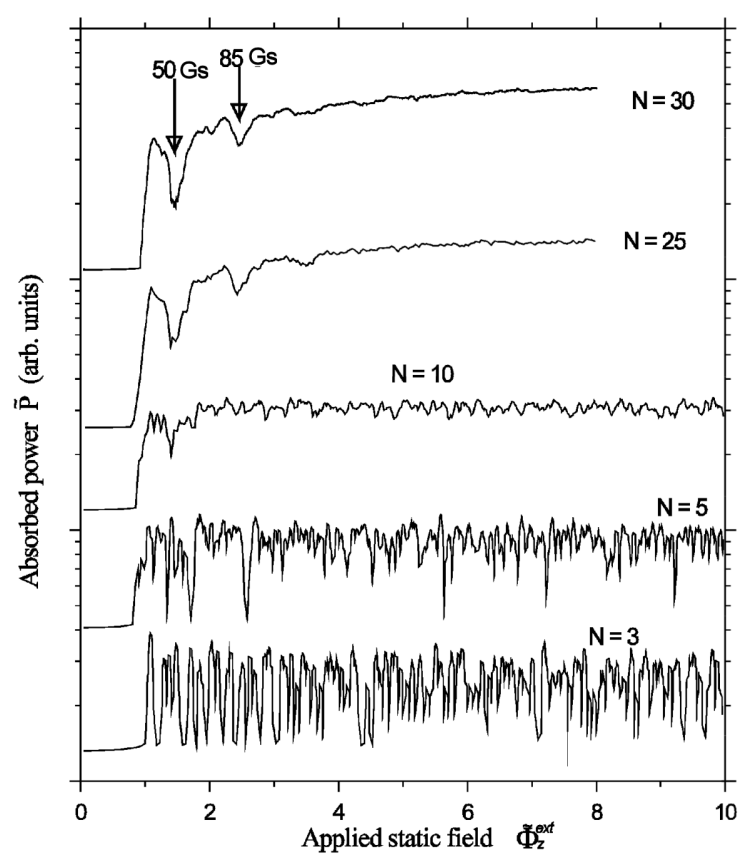

Fig. 3. Power absorption vs. applied magnetic field for the network containing $N \times N \times N$ junctions; the parameters $\tilde{\Gamma}$ and $\tilde{L}$ where undergoing $10 \%$ Gaussian fluctuations around the mean values $\tilde{\Gamma}_{0}=5$ and $\tilde{L}_{0}=1$.

sionless units, where $U$ is the voltage drop on resistance $R$. The representative shape of the applied field dependence (in units $\Phi / \Phi_{0}$ ) of $\tilde{P}$ is displayed in Fig. 3 for several values of $N=3-30$. We see that the discrete absorption peaks average out with increasing $N$. Nonetheless, two characteristic features survive. First of them is that the absorption starts at the value $\tilde{\Phi}_{z}^{\text {ext }}=0.9$ (corresponding to $10 \%$ reduction of the elementary loop size from the standard value equal to unity). Second, well-defined minima develop at specific values of $\tilde{\Phi}_{z}^{\text {ext }}$. These minima are also present on the experimental curve (cf. Fig. 1, where the derivative $\mathrm{d} P / \mathrm{d} \Phi_{z}$ was measured). The first minimum is positioned at $\tilde{\Phi}_{z}^{\text {ext }}=1.4$, which in combination 
with the average grain size $d=0.74 \mu \mathrm{m}$ gives the magnetic induction $B_{1}=50$ Gs in an excellent agreement with the experiment. The second minimum is placed at $\tilde{\Phi}_{z}^{\text {ext }}=2.4$, which leads to the induction value $B_{2}=85$ Gs.

The described effect is more pronounced with increasing $N$ and must be common to all inhomogeneous superconductors when the statistic improves. It is related to the dispersion in size distribution of the crystallites and should take place only if the statistical distribution is peaked around one or two sizes (in the second case it means that we have bicrystallites coexisting with the crystallites). To understand these secondary features in detail we have to analyze the array with variable $N$ and identical physical parameters. The results of the simulation

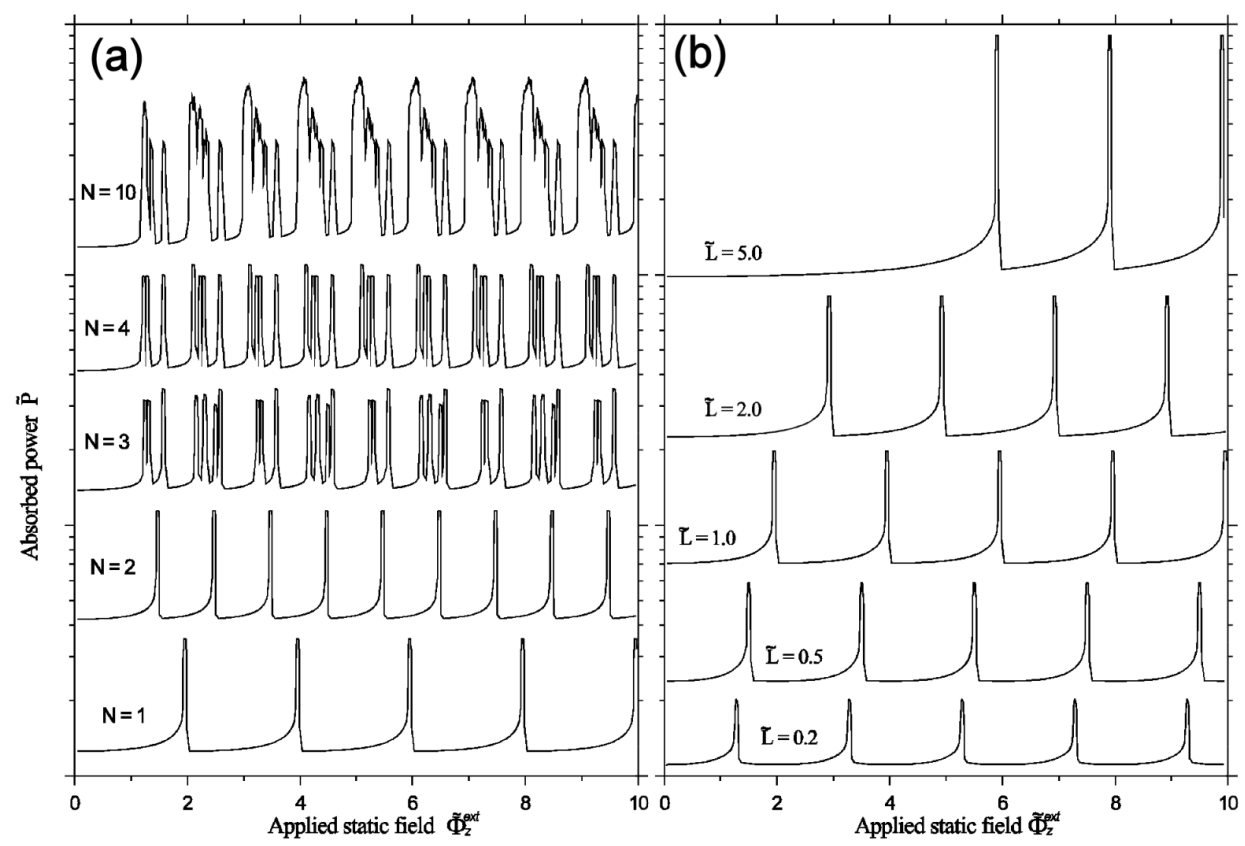

Fig. 4. Applied field dependence of the microwave power absorption for (a) different networks of identical Josephson junctions $(\tilde{\Gamma}=5, \tilde{L}=1)$, and (b) a cube containing 12 junctions $(\Gamma=5$ and different $\tilde{L})$.

for such network containing $N \times N \times N$ junctions are displayed in Fig. 4a, for the values of parameters $N=1 \div 10, \tilde{\Gamma}=5$ and $\tilde{L}=1$. With increasing $N$ we observe the systematic filling of the space between the discrete resonance (physical discussion of which is provided below), which eventually for $N \rightarrow \infty$ smoothens out the absorption curve above the threshold value of $\tilde{\Phi}_{z}^{\text {ext }}=1$. The situation with a lack of sizable statistical variations of the junction parameters may explain the recently observed [11] smooth variation of the power absorption in $\mathrm{YBaCuO}$ samples. 


\subsection{Physical discussion}

The first simulation cycle of the absorption was carried out on a single cube containing 12 junctions without random variation of the parameters. Strictly speaking, we have studied the evolution of the absorption maxima with increasing inductance $\tilde{L}$. Elementary analysis suggests that the first absorption maximum appears for a single junction at $\tilde{\Phi}_{z}^{\text {ext }}=\tilde{L}$, which determines the connection between $\tilde{L}$ and the lower critical field for the sample

$$
B_{\mathrm{c} 1}=\frac{\tilde{L} \Phi_{0}}{d^{2}}
$$

This result follows from the circumstance that for $\tilde{\Phi}_{z}^{\text {ext }}=\tilde{L}$ the current screening the external flux in $x y$ plane achieves a critical value for the appearance of nonzero voltage. The results presented in Fig. 4b prove that the maxima in question appear at higher field depending on inductance. The presence of the microwave field does not account for the difference, as it shifts the maximum in the opposite way.

The qualitative analysis of the differential equations describing the system helps to explain the maxima positions in the following manner. Let us consider a planar $(x y)$ loop containing 4 junctions in the static field $\tilde{\Phi}_{z}^{\text {ext }}$. Because of symmetry Eqs. (9) and (3) (with the condition (10)) reduce to the form

$$
\ddot{\tilde{\theta}}+\tilde{\Gamma} \dot{\tilde{\theta}}+\tilde{L} \sin 2 \pi \tilde{\theta}=-4 \tilde{\theta}-\tilde{\Phi}_{z}^{\text {ext }},
$$

or equivalently

$$
\ddot{\tilde{\theta}}+\tilde{\Gamma} \dot{\tilde{\theta}}=-\frac{\mathrm{d}}{\mathrm{d} \tilde{\theta}}\left[2\left(\tilde{\theta}+\frac{1}{4} \tilde{\Phi}_{z}^{\text {ext }}\right)^{2}-\frac{\tilde{L}}{2 \pi} \cos 2 \pi \tilde{\theta}\right] .
$$

The last equation describes damped oscillations in a potential field

$$
V(\tilde{\theta})=2\left(\tilde{\theta}+\frac{1}{4} \tilde{\Phi}_{z}^{\mathrm{ext}}\right)^{2}-\frac{\tilde{L}}{2 \pi} \cos 2 \pi \tilde{\theta}
$$

For the absence of applied field the system has a minimum at rest: $\tilde{\theta}=0$. With increasing $\tilde{\Phi}_{z}^{\text {ext }}$ the equilibrium position shifts until the minimum becomes an inflection point, at which a catastrophe of $A_{2}$ type occurs. The situation is represented schematically in Fig. 5. The minima at the $V(\tilde{\theta})$ curves (cf. Fig. 5a) are determined by the condition

$$
4 \tilde{\theta}+\tilde{L} \sin 2 \pi \tilde{\theta}=-\tilde{\Phi}_{z}^{\text {ext }} .
$$

For large $\tilde{L}$ this equation has many solutions, but starting from $\tilde{\theta}=0$ for $\tilde{\Phi}_{z}^{\text {ext }}$ we can establish unambiguously, which is realized, as illustrated in Fig. 5b. From this figure we see that the catastrophes take place for the $\tilde{\theta}$ values, for which the function

$$
f(\tilde{\theta})=4 \tilde{\theta}+\tilde{L} \sin 2 \pi \tilde{\theta}
$$

acquires minima, i.e. for

$$
\tilde{\theta}=\tilde{\theta}_{\mathrm{c}}=-\frac{1}{4}-\frac{1}{2 \pi} \arcsin \frac{2}{\pi \tilde{L}}+k,
$$




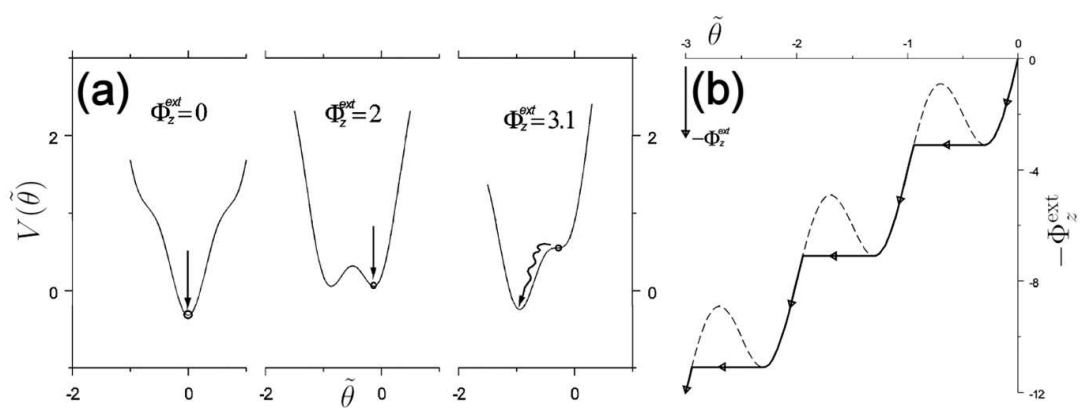

Fig. 5. Effective potential $V(\tilde{\theta})$ for a square loop composed of 4 Josephson junctions: (a) Evolution with an applied field, the arrow points to the equilibrium state. (b) Solution of Eq. (17) for $\tilde{\theta}=0$ and $\tilde{\Phi}_{z}^{\text {ext }}=0$.

where $k=0,1,2, \ldots$ The values $\tilde{\theta}_{\mathrm{c}}$ lead to the values of the flux

$$
\tilde{\Phi}_{z}^{\mathrm{ext}}=\tilde{\Phi}_{\mathrm{c}}\left(A_{\mathrm{m}}=0\right)=-f\left(\tilde{\theta}_{\mathrm{c}}\right)=1+\frac{2}{\pi} \arcsin \frac{2}{\pi \tilde{L}}+\tilde{L} \sqrt{1-\frac{4}{\pi^{2} \tilde{L}^{2}}}+4 k .
$$

This expression determines approximately only every second absorption maximum position displayed in Fig. 4b. This is because we have ignored the effect of the microwave field, which is parallel to the $x$-axis, which breaks the equivalence between $x$ - and $y$-axes. An additional simulation has shown that even for a very small amplitude $A_{\mathrm{m}}=10^{-15}$ of the microwave field the picture is changed drastically: the number of maxima doubles and their positions shift by a small jump. An additional problem is connected with the fact that Eq. (20) is not defined for $\tilde{L}<2 / \pi$, a circumstance leading to a continuous evolution. A detailed analysis of stability of the system of differential equations describing the discussed 12 junctions is quite lengthy and after a linearization with respect to $A_{\mathrm{m}}$ leads to the following expression replacing (20) (see Appendix):

$$
\tilde{\Phi}\left(A_{\mathrm{m}} \rightarrow 0\right)=1+\frac{2}{\pi} \arcsin \frac{1}{\pi \tilde{L}+1}+\tilde{L} \sqrt{1-\frac{1}{(\pi \tilde{L}+1)^{2}}}+2 k .
$$

This approximate analytic result is compared with the simulations in Table. The agreement is quite good, particularly for large $\tilde{L}$.

Stability analysis complicates enormously for large $N$, even for $A_{\mathrm{m}}=0$. However, we can grasp the difference between the results for $N=1$ and $N=2$ in Fig. 4a. Namely, taking for $N=2$ the problem symmetry then we can reduce effectively our reasoning to the $N=1$ situation by merely rescaling the quantities: $\tilde{\theta} \rightarrow 2 \tilde{\theta}, \tilde{\Phi} \rightarrow 4 \tilde{\Phi}$, and $\tilde{L} \rightarrow 4 \tilde{L}$. This explains the doubling of the number of maxima when moving from $N=1$ to $N=2$ case. The existence of additional maxima for $N>2$ in Fig. 4a indicates the presence of other oscillation modes leading to the subsidiary maxima near the main maxima. For $N=10$ we observe already an absorption band widening with growing system size. Additionally, for $N>2$ a gradual growth of maxima in the lowest regime of $\tilde{\Phi}_{z}^{\text {ext }}$ takes place. The 


\section{TABLE}

Self-inductance dependence of the first absorption maximum position for a cube composed of 12 Josephson junctions (obtained from Eq. (21)), and compared with the computed values for a very small $\left(10^{-15}\right)$ and realistic $(0.1)$ amplitudes of the microwave field.

\begin{tabular}{c|c|c|c}
\hline \hline$\tilde{L}$ & Eq. (21) & $A_{\mathrm{m}}=10^{-15}$ & $A_{\mathrm{m}}=0.1$ \\
\hline 0.2 & 1.579 & 1.616 & 1.276 \\
0.5 & 1.715 & 1.733 & 1.489 \\
1.0 & 2.126 & 2.153 & 1.935 \\
2.0 & 3.069 & 3.093 & 2.906 \\
5.0 & 6.029 & 6.032 & 5.882
\end{tabular}

absorption maxima become periodic with the interval of one quantum (i.e. $\tilde{\Phi}=1$ ) only for $\tilde{\Phi}_{z}^{\text {ext }}>4$ for $\tilde{L}=1$. This phenomenon seems to be analogous to the existence of two critical fields in bulk samples.

The character change of the absorption spectrum obtained after introducing the random fluctuations of the junction parameters is also easy to grasp quantitatively. Each discrete absorption line for a regular array depicted in Fig. 4 is convoluted with the probability distribution. The lines for large $\tilde{\Phi}_{z}^{\text {ext }}$ are smeared out proportionally in a wide region, so their sum will result in a practically constant value of the absorption. The expression (13) for the first critical field is valid; that is why one can use it to translate the dimensionless simulation parameters onto physical units [9]. This is simply due to the fact that for $\tilde{\Phi}_{z}^{\text {ext }}<\tilde{L}$ absorption is zero, since the superconducting loops screen the external field.

As we have seen, the absorption effects are amplified with growing $N$. This proves that they are common to all inhomogeneous superconductors, in distinction to the dispersion of results in one simulation cycle, which disappears with the better statistics.

\section{Summary}

A general method has been presented of generating a system of second-order differential equations, which describe the time evolution of three-dimensional Josephson networks with inclusion of their resistivity, inductance, and capacity. The effect of junction capacity is important, since we include high frequency microwave field. Apart from a characteristic field $B_{\mathrm{c} 1}$ we observe the existence of second critical field described above.

The analysis of the stability of those equations for a single structural cell (a cube containing 12 junctions) provides the absorption maxima positions only after inclusion of the small microwave field amplitude. Also, the networks of the 
size $30 \times 30 \times 30$ and with a $10 \%$ random variations of the junction parameters are sufficient to account for the experimental observations.

\section{Acknowledgments}

The authors acknowledge the support by the Ministry of Science, grant No. 1 P03B 001 29. A.R. acknowledges a special grant of Polish Science Foundation (FNP). The discussion with our colleagues: Prof. A. Kołodziejczyk, J. Niewolski and Prof. J. Stankowski are appreciated.

\section{Appendix}

\section{Stability of the system of differential equations} for the cubic elementary cell of junctions

In this Appendix we derive the analytic result (21) from the stability considerations for the system of differential equations, describing the time evolution of the phases. The reasoning is carried out for an isolated structural unit composed of 12 Josephson junctions (an elementary cube) with inclusion of resistances and capacitances. This cell is placed in the applied field static parallel to $z$-axis and the microwave field parallel to $x$-axis. Then, the symmetry of the problem reduces to consideration of four invariant phases $\theta^{x}, \theta^{z}, \theta_{1}^{y}$, and $\theta_{2}^{y}$, as well as of two mag-

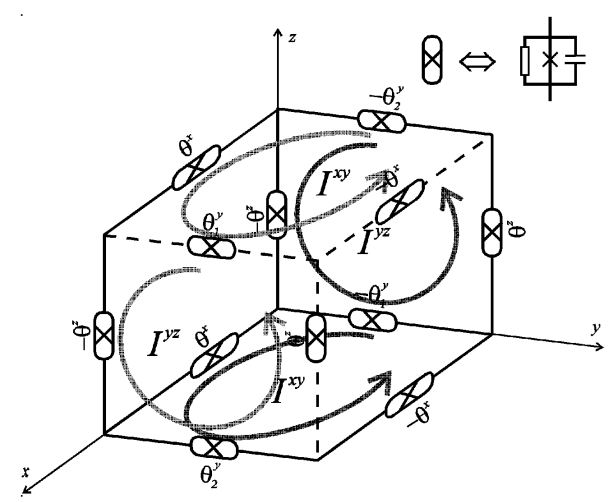

Fig. 6. Isolated cubic elementary cell composed of 12 Josephson junctions, together with the corresponding independent gauge invariant phases and currents. The independent magnetic fluxes reflect the marked currents.

netic fluxes $\Phi^{x y}$ and $\Phi^{y z}$, and to the loop current $I^{x y}$ and $I^{y z}$. The situation is depicted schematically in Fig. 6. The magnetic fluxes can be related to the phase differences and the currents in the following manner (cf. Eqs. (8)):

$$
\begin{aligned}
& \tilde{\Phi}^{x y}=-\left(\tilde{\theta}_{2}^{y}+\tilde{\theta}_{1}^{y}+2 \tilde{\theta}^{x}\right)=\tilde{\Phi}_{z}^{\mathrm{ext}}+\tilde{L} \tilde{I}^{x y} \\
& \tilde{\Phi}^{y z}=-\left(\tilde{\theta}_{2}^{y}-\tilde{\theta}_{1}^{y}+2 \tilde{\theta}^{z}\right)=\tilde{\Phi}_{x}^{\mathrm{ext}}+\tilde{L} \tilde{I}^{y z}
\end{aligned}
$$


where $\tilde{\Phi}_{x}^{\text {ext }}$ and $\tilde{\Phi}_{z}^{\text {ext }}$ are the fluxes parallel to the corresponding axes (in units of $\left.\Phi_{0}\right)$.

The dynamic Eqs. (9) take the form

$$
\begin{aligned}
& \ddot{\tilde{\theta}}^{x}+\tilde{\Gamma} \dot{\tilde{\tilde{\theta}}}^{x}+\tilde{L} \sin 2 \pi \tilde{\theta}^{x}=\tilde{L} \tilde{I}^{y z}, \\
& \ddot{\tilde{\theta}}_{1}^{y}+\tilde{\Gamma} \dot{\tilde{\theta}}_{1}^{y}+\tilde{L} \sin 2 \pi \tilde{\theta}_{1}^{y}=\tilde{L}\left(\tilde{I}^{x y}-\tilde{I}^{y z}\right), \\
& \ddot{\tilde{\theta}}_{2}^{y}+\tilde{\Gamma} \dot{\tilde{\theta}}_{2}^{y}+\tilde{L} \sin 2 \pi \tilde{\theta}_{2}^{y}=\tilde{L}\left(\tilde{I}^{x y}+\tilde{I}^{y z}\right), \\
& \ddot{\tilde{\theta}}^{z}+\tilde{\Gamma} \dot{\tilde{\theta}}^{z}+\tilde{L} \sin 2 \pi \tilde{\theta}^{z}=\tilde{L} \tilde{I}^{x y} .
\end{aligned}
$$

Adding and subtracting second and third equations in (23), and substituting expressions for $\tilde{L} \tilde{I}^{x y}$ and $\tilde{L} \tilde{I}^{y z}$ calculated from (22), we obtain

$$
\begin{aligned}
& \ddot{\tilde{\theta}}^{x}+\tilde{\Gamma} \dot{\tilde{\theta}}^{x}+\tilde{L} \sin 2 \pi \tilde{\theta}^{x}=-2 \tilde{\vartheta}_{+}-2 \tilde{\theta}^{x}-\tilde{\Phi}_{z}^{\mathrm{ext}} \\
& \ddot{\tilde{\vartheta}}_{+}+\tilde{\Gamma} \dot{\tilde{\vartheta}}_{+}+\tilde{L} \cos 2 \pi \tilde{\vartheta}_{-} \sin 2 \pi \tilde{\vartheta}_{+}=-2 \tilde{\vartheta}_{+}-2 \tilde{\theta}^{x}-\tilde{\Phi}_{z}^{\mathrm{ext}} \\
& \ddot{\tilde{\vartheta}}_{-}+\tilde{\Gamma} \dot{\tilde{\vartheta}}_{-}+\tilde{L} \cos 2 \pi \tilde{\vartheta}_{+} \sin 2 \pi \tilde{\vartheta}_{-}=-2 \tilde{\vartheta}_{-}-2 \tilde{\theta}^{z}-\tilde{\Phi}_{x}^{\mathrm{ext}} \\
& \ddot{\tilde{\theta}}^{z}+\tilde{\Gamma} \dot{\tilde{\theta}}^{z}+\tilde{L} \sin 2 \pi \tilde{\theta}^{z}=-2 \tilde{\vartheta}_{-}-2 \tilde{\theta}^{z}-\tilde{\Phi}_{x}^{\mathrm{ext}}
\end{aligned}
$$

where the new variables are defined as

$$
\tilde{\vartheta}_{+} \equiv \frac{1}{2}\left(\tilde{\theta}_{2}^{y}+\tilde{\theta}_{1}^{y}\right), \quad \tilde{\vartheta}_{-} \equiv \frac{1}{2}\left(\tilde{\theta}_{2}^{y}-\tilde{\theta}_{1}^{y}\right)
$$

The last two equations in (24) describe damped oscillations of two coupled nonlinear oscillators under the influence of the external force

$$
\underline{F}=\left(\begin{array}{c}
F_{\tilde{\vartheta}_{-}} \\
F_{\tilde{\theta}^{z}}
\end{array}\right), \quad F_{\tilde{\vartheta}_{-}}=F_{\tilde{\theta}^{z}}=-\tilde{\Phi}_{x}^{\text {ext }}
$$

and in the potential

$$
\begin{aligned}
& V\left(\tilde{\vartheta}_{-}, \tilde{\theta}^{z}\right)=-\frac{L \cos 2 \pi \tilde{\vartheta}_{+}}{2 \pi} \cos 2 \pi \tilde{\vartheta}_{-} \\
& +\left(\tilde{\vartheta}_{-}\right)^{2}+2 \tilde{\vartheta}_{-} \tilde{\theta}^{z}+\left(\tilde{\theta}^{z}\right)^{2}-\frac{\tilde{L}}{2 \pi} \cos 2 \pi \tilde{\theta}^{z} .
\end{aligned}
$$

We show below that the $\tilde{\vartheta}_{+}$dependence of the potential is connected to $\tilde{\Phi}_{z}^{\text {ext }}$ and thus vanishes if $\tilde{\Phi}_{z}^{\text {ext }}=0$. Therefore, $V\left(\tilde{\vartheta}_{-}, \tilde{\theta}^{z}\right)$ has a sharp minimum at $\tilde{\vartheta}_{-}=\tilde{\theta}^{z}=0$. Furthermore, since along the $x$-axis $\tilde{\Phi}_{x}^{\text {ext }}=A_{\mathrm{m}} \sin (2 \pi \tilde{t} / T)$, with $A_{\mathrm{m}} \ll 1$, and the force is given by (26), we have for small vibrations around the minimum that

$$
V\left(\tilde{\vartheta}_{-}, \tilde{\theta}^{z}\right) \cong \frac{1}{2}\left(\tilde{\vartheta}_{-} \tilde{\theta}^{z}\right)\left(\begin{array}{cc}
2 \pi \tilde{L} \cos 2 \pi \tilde{\vartheta}_{+}+2 & 2 \\
2 & 2 \pi \tilde{L}+2
\end{array}\right)\left(\begin{array}{c}
\tilde{\vartheta}_{-} \\
\tilde{\theta}^{z}
\end{array}\right) .
$$

The instability threshold of the system (24) is reached when the quadratic 
form (28) ceases to be positively determined. This happens for

$$
\tilde{\vartheta}_{+}=-\frac{1}{4}-\frac{1}{2 \pi} \arcsin \frac{1}{\pi \tilde{L}+1}
$$

It remains still to determine the relation between $\tilde{\vartheta}_{+}$and $\tilde{\Phi}_{z}^{\text {ext }}$. Since $A_{\mathrm{m}} \ll 1$, then we can set at $t=0, \tilde{\Phi}_{z}^{\text {ext }}=0$; then $\tilde{\theta}_{1}^{y} \cong \tilde{\theta}_{2}^{y} \cong \tilde{\theta}^{x}$, and hence

$$
\tilde{\vartheta}_{+} \cong \tilde{\theta}^{x}, \quad \tilde{\vartheta}_{-} \cong 0 \text {. }
$$

Thus, the relation to $\tilde{\Phi}_{z}^{\text {ext }}$ can be determined by considering the equilibrium points, for which e.g. the first equation of (24) reduces to the form $\tilde{L} \sin 2 \pi \tilde{\theta}^{x}+2 \tilde{\vartheta}_{+}+\tilde{\theta}^{x}=$ $-\tilde{\Phi}_{z}^{\text {ext }}$. Taking into account (30) and (29) we obtain the critical flux value

$$
\tilde{\Phi}_{\mathrm{c}}=1+\frac{2}{\pi} \arcsin \frac{1}{\pi \tilde{L}+1}+\tilde{L} \sqrt{1-\frac{1}{(\pi \tilde{L}+1)^{2}}} .
$$

This formula determines the applied field value, for which the first catastrophe takes place. Formally, the critical value of $\tilde{\vartheta}_{+}$, for which the determinant of the quadratic form (28) vanishes, repeats every unit. In effect, the critical value $\Phi_{\mathrm{c}}$ should repeat itself every 4 units. This does not happen because at the catastrophe, at which (27) changes locally from paraboloidal into saddle-like, the small oscillation approximation loses its meaning. The $\tilde{\vartheta}_{-}$value does not grow without limit but changes abruptly by the amount $\pm \frac{1}{2}$, i.e. the system jumps to the closest minimum, where again we can use for the time being the small-oscillation considerations. The sign is not important. Nonetheless, such change of $\tilde{\vartheta}_{-}$determines the sign change of the term $\cos \left(2 \pi \tilde{\vartheta}_{-}\right)$in the second equation of (24) system. Therefore, the equilibrium condition for $\tilde{\theta}^{x}$ and $\tilde{\vartheta}_{+}$has now the form

$$
\tilde{L} \sin 2 \pi \tilde{\theta}^{x}+2 \tilde{\vartheta}_{+}+2 \tilde{\theta}^{x}=-\tilde{\Phi}_{z}^{\text {ext }}, \quad-\tilde{L} \sin 2 \pi \tilde{\vartheta}_{+}+2 \tilde{\vartheta}_{+}+2 \tilde{\theta}^{x}=-\tilde{\Phi}_{z}^{\text {ext }},
$$

from which we have $\sin 2 \pi \tilde{\theta}^{x}+\sin 2 \pi \tilde{\vartheta}_{+}=0$, and thus, the magnitude of the jump is $\tilde{\vartheta}_{+} \cong \tilde{\theta}^{x} \rightarrow \tilde{\vartheta}_{+} \cong \tilde{\theta}^{x} \pm \frac{1}{2}$. This means that at each catastrophe the system overcomes, at given magnetic field, half of the way to the next critical point (or pulls back by the same value). This, in turn leads to the frequency doubling, with which catastrophes happen with a slow increase in static external field. In effect this leads to

$$
\tilde{\Phi}_{\mathrm{c}}=1+\frac{2}{\pi} \arcsin \frac{1}{\pi \tilde{L}+1}+\tilde{L} \sqrt{1-\frac{1}{(\pi \tilde{L}+1)^{2}}}+2 k,
$$

where $k=0,1,2, \ldots$ This is formula (21) in the main text.

\section{References}

[1] J. Stankowski, P.K. Kahol, N.S. Dalal, J.S. Moodera, Phys. Rev. B 36, 7126 (1987). For a theoretical discussion in terms of a single junction see: B. Czyżak, Physica C 243, 327 (1995) and references therein.

[2] R. Durny, J. Hantela, S. Ducharme, B. Lee, O.G. Symko, P. Taylor, D.Z. Zheng, J.A. Xu, Phys. Rev. B 36, 2361 (1987). 
[3] K.W. Blazey, K.A. Müler, J.G. Bednorz, W. Berlinger, G. Amoretti, E. Buluggiu, A. Vera, F.C. Matacotta, Phys. Rev. B 36, 7241 (1987).

[4] C. Jeffries, Q.H. Lam, Y. Kim, L.C. Bourne, A. Zettl, Phys. Rev. B 39, 4792 (1989); Ting-Kang Xia, D. Stroud, Phys. Rev. B 39, 4792 (1989).

[5] H. Vichery, F. Beuneu, P. Lejay, Physica C 159, 823 (1989).

[6] For general introduction see: A. Barone, G. Paterno, Physics and Application of the Josephson Effect, Wiley, New York 1982; M. Tinkham, C.J. Lobb, in: Solid State Physics, Vol. 42, Eds. H. Ehrenreich, D. Turnbull, Academic, San Diego 1989, p. 91.

[7] See e.g. J.R. Clem, Physica $C$ 162-164, 1137 (1989) and references therein; T. Wolf, A. Majhofer, Phys. Rev. B 47, 5383 (1993); D. Reinel, W. Dietrich, A. Majhofer, T. Wolf, Physica C 245, 193 (1995).

[8] W. Lawrence, S. Doniach, in: Proc. Twelfth Int. Conf. on Low Temperature Physics, Ed. E. Kanda, Academic Press of Japan, Kyoto 1971, p. 361.

[9] A. Rycerz, J. Spałek, Physica C 387, 97 (2003).

[10] R.L. Burden, J.D. Faires, Numerical Analysis, Prindle, Weber \& Schmidt, Boston 1985, p. 220.

[11] J. Niewolski, A. Kołodziejczyk, W.M. Woch, Mol. Phys. Rep. (Poland) 20, 199 (1997); K. Przybylski, W.M. Woch, A. Kołodziejczyk, J. Niewolski, Superlattices Microstruct. 21 (Suppl. A), 169 (1997), and unpublished. 